REVIEW

\title{
Oral mucositis induced by anticancer treatments: physiopathology and treatments
}

\author{
D'Hondt, Lionel \\ Lonchay, Christophe \\ André, Marc \\ Canon, Jean-Luc \\ Centre Hospitalier Notre-Dame et \\ Reine Fabiola, Charleroi, Belgium
}

\begin{abstract}
Oral mucositis is a frequent and devastating side effect of anticancer treatments. It impairs the patient's quality of life and also can be life threatening because severe infections and delayed or incomplete anticancer treatments may result. This problem has been largely overlooked and underestimated in the past. However, recently studies have been performed to precisely identify the epidemiology, cost, consequences, physiopathology, and treatments of oral mucositis. Clinical guidelines have recently been published to help the daily management of this frequent complication. In addition, some innovative new drugs, including palifermin, have been developed to prevent and treat this major side effect of cancer treatments. In this paper we summarize the recent developments of oral mucositis management.
\end{abstract}

Keywords: chemotherapy, radiotherapy, oral mucositis, palifermin

\section{Introduction}

Anticancer treatment-related mucosal damage to the oral cavity is referred to as oral mucositis, and until recently it has been underestimated and overlooked (Wright et al 2005). Treatments of this important side effect have likely been suboptimal and based on assumptions rather than evidence. In addition, physiopathologic aspects have not been addressed. Fortunately, this important issue has received greater attention in recent years because oral mucositis not only has a dramatic impact on the patient's quality of life, but it also can adversely influence the administration of an optimal antineoplasic treatment. Frequently, dose reductions, treatment delays, or discontinuations are necessary to allow the oral lesions to heal. In addition, lifethreatening infections and higher treatment costs are clearly related to the severity of oral mucositis. Additional days of total parenteral nutrition, use of narcotic analgesics, total hospital charges, and 100-day mortality are also linked to the severity of mucositis (Rubenstein et al 2004).

Important epidemiologic studies have been conducted in an attempt to isolate risk factors and a great deal of basic research has advanced our understanding of the mechanisms underlying oral mucositis. Taken together, these findings have provided the basis for the development of new target therapies. Recently there have been efforts to summarize knowledge acquired in the treatment of stomatitis. A critical review of the literature allowed a panel of experts to propose clinical practice guidelines for the prevention and treatment of this complication (Rubenstein et al 2004; Sonis et al 2004). This panel of experts from the Multinational Association of Supportive Care and the International Society for Oral Oncology (MASSC/ISOO) formed the Mucositis Study Section. In this paper, we will focus on anticancer-induced oral mucositis, detailing the physiopathology and the treatment of this devastating complication. We will particularly emphasize the emerging role of palifermin (recombinant human keratinocyte growth factor), the first US Food and Drug Administration (FDA)-approved target therapy for oral mucositis. 


\section{Epidemiology}

Cancer patients consider oral mucositis to be the most difficult treatment-related toxicity to endure. The frequency of oral mucositis is about $30 \%$ to $40 \%$ in the general cancer patient population (Sonis 2004). However, patients receiving highdose chemotherapy (HDC) conditioning before hematopoietic stem cell transplant (HSCT) have a 76\% chance of developing mucositis (Rao Naidu et al 2004). In addition, patients receiving radiation, especially for head-and-neck cancers, have a $30 \%$ to $60 \%$ chance of suffering from this complication. The increased incidence is not only related to drugs used but also to the number of chemotherapy cycles and the previous occurrence of mucositis (Rao Naidu et al 2004). It is estimated that annually there are approximately 400000 cases of therapyinduced oral mucositis (Rao Naidu et al 2004). Risk factors can be divided into two main categories: risks related to patient status and those related to the treatment itself.

\section{Patient-related risks}

Age has been shown to be a risk factor because children experience more frequent and debilitating mucositis, probably because of high cellular turnover. In addition, patients over 50 years of age may develop severe mucositis, perhaps because of insufficient DNA repair (Balducci and Exterrman 2000). Female patients are more likely to develop oral mucositis, for instance after 5-fluorouracil (5-FU) treatment (Sonis 1998). Nutritional status, type of malignancy, drug-induced xerostomia (dry mouth), pre-existing mouth damage, and periodontal status also play a role. Tobacco and alcohol consumption is probably an important risk factor, although this remains controversial (Dood et al 1999).

In addition, there has been a recent focus on genetic predisposition for oral mucositis. Enzymatic phenotypes probably play a key role in explaining the huge variation of interindividual tolerance to anticancer treatment. For instance, Ulrich et al (2001) reported that in patients undergoing HDC for HSCT, a polymorphism in the 5, 10-methylenetetrahydrofolate reductase gene was associated with a differential rate of oral mucositis after treatment with methotrexate. Finally, previous episodes of oral mucositis are also an important risk factor.

\section{Treatment-related risk}

Mucositis is the second most frequent dose-limiting factor in patients receiving chemotherapy (Peterson and Cariello 2004). Chemotherapeutic drugs affecting DNA synthesis are particularly stomatotoxic. For instance, 5-FU administration is associated with oral mucositis in about $40 \%$ of patients
(Popescu et al 1999), and grade 3 to 4 oral mucositis is observed in $10 \%$ to $15 \%$ of 5 -FU recipients (MAC 1998). There is an increased risk of mucositis with bolus and continuous infusions compared with prolonged and repetitive low doses of chemotherapeutic agents (Rao Naidu et al 2004). Other cytotoxic agents are known to be very damaging to mucosa (eg, alkylating agents such as anthracyclines and taxanes and folate-based drugs such as methotrexate) (Sharma et al 2005; Wright et al 2005). Finally, some drugs are excreted in the saliva, such as methotrexate and etoposide (Rao Naidu et al 2004).

The dose of chemotherapy is also of great importance, as illustrated by the $85 \%-95 \%$ incidence of mucositis in HSCT recipients. Conditioning regimens including melphalan are associated with high rates of oral mucositis (Wardley et al 2000). In this setting, mucositis has been reported by patients as the most distressing side effect they had to cope with (Bellm et al 2000). It is interesting to note that allogeneic transplant recipients experience higher grades of mucositis compared with those receiving autologous transplants (Wright et al 2005). In addition, there is a strong relationship between low neutrophilic count and the severity of stomatitis (Saadeh 2005).

Radiotherapy is also associated with an increased risk of mucositis. The risk varies with dose, fractioning, and the site of radiotherapy. In head-and-neck cancer patients, the incidence of mucositis is over $50 \%$, and this rate increases when chemotherapy is given concomitantly or in HSCT recipients when total body irradiation is administered (Trotti et al 2000; Rubenstein et al 2004). Accelerated fraction even increases the risk of mucositis to $>70 \%$, at which point mucositis is often dose limiting (Trotti et al 2000). A summary of the different risk factors is presented in Table 1 .

The severity of mucositis has a direct influence on the treatment planning with the necessity of dose reductions, delays, or even discontinuations of anticancer therapy. This condition also can favor the development of life-threatening infections, especially if the patient is neutropenic. We recently reported the case of a head-and-neck cancer patient with extensive stomatitis who developed a Saccharomyces cerevisiae fungemia infection during concomitant chemoradiotherapy (Henry et al 2004).

Oral mucositis has important economic costs. In a recent economic study, patients with mucositis had significantly higher costs for nutrition/hydration support and prescription medications but not for hospitalization compared with headand-neck cancer patients free of mucositis. This difference resulted, however, in higher total costs for patients with mucositis (Peterman et al 2001). 
Table I Proven and suspected risk factors for the development of mucositis

Risk factors for the development of mucositis

Patient-related risk factors
Age: Children and over 50 years old
Female sex
Denutrition
Tumor location (eg, oral cavity, throat)
Drug-induced xerostomia
Pre-existing mouth damage
Periodontal status
Tobacco and alcohol consumption
Genetic predisposition

Finally, it should be noted that there are huge discrepancies in the way the severity of mucositis is described. Different scales exist; the most commonly used are the National Cancer Institute - Common Toxicity Criteria and the scale developed by the World Health Organization (Sonis et al 2004). These scales are graded from 0 to 4 , and they are highly reproducible and easy to use, combining evaluation of objective functions and symptomatic variables. Grades III and IV are the most debilitating states because patients are no longer able to eat or require parenteral or enteral support. Other scales have been developed, but they are essentially used for research purposes. All of these methods of evaluation have been extensively reviewed by Sonis et al (2004) and Peterson and Cariello (2004). Recently, interesting pathologic evaluations also have been described. These evaluations result in, for example, estimates of the cell viability after mouthwashes using trypan blue coloration (Wynenga et al 1999), measurement of neutrophil levels in mouthwashes (Graham et al 1992), or assessment of epithelial cell morphology, and differentiation/ maturation (Wynenga et al 1997). The use of these in vitro pathological tests must be encouraged because they can reduce the bias of subjective methodology.

\section{Physiopathology}

Clinically, oral mucositis appears about 7 to 10 days after the start of chemotherapy. Recovery occurs between days 14 to 21 (Saadeh 2005). However, mucositis complicating radiotherapy usually develops later, and it has a more chronic course (Sonis et al 2004). It starts at a cumulative dose of 15 Grays (Gy) and peaks at 30 Gy. Despite this difference in timing of occurrence, physiopathologic mechanisms underlying the
Treatment-related risk factors

$\begin{array}{ll}\text { Chemotherapy: } & \begin{array}{l}\text { Type of drug } \\ \text { Dose and intensity } \\ \text { Induced neutropenia }\end{array} \\ \text { Radiotherapy: } & \begin{array}{l}\text { Fractioning } \\ \text { Location } \\ \text { Combined with chemotherapy }\end{array} \\ \text { Bone marrow transplantation }\end{array}$

Bone marrow transplantation development of oral mucositis are probably identical, and these mechanisms have recently been thoroughly explored. It has become clear now that oral mucositis is not only the direct consequence of a direct physical or chemical epithelial damage but also the result of a cascade of events involving the entire mucosa (Sonis 2004). Recently, Sonis and co-workers (2004) proposed an interesting model that describes the different biological events occurring during mucositis. In this model, five different but interdependent phases have been identified, as described below and depicted in a flow chart in Figure 1.

\section{Initiation}

Initiation is the first phase. Immediately after exposure to radiotherapy or chemotherapy, there is DNA and non-DNA damage. This damage is observed in mucosa and submucosa, but only a small proportion of cells die quickly (Sonis 2004). However, this initial insult triggers a cascade of biological events. Generation of oxidative stress and reactive oxygen species is thought to be the origin of injuries to mucosal cells, tissues, and blood vessels.

\section{Message generation}

Message generation is the second phase. During this period, there is an upregulation of transcription factors, such as nuclear factor- $\kappa \mathrm{B}(\mathrm{NF}-\kappa \mathrm{B})$, and genes coding for proinflammatory cytokines (eg, tumor necrosis factor [TNF- $\alpha$ ], and interleukins [ILs] IL-1, IL2, and IL-6) and adhesion molecules. In addition, enzymes such as neutral and acidic sphingomyelinases and ceramide synthase are activated. This activation results in apoptosis of submucosal endothelial cells and fibroblasts. Fibroblast destruction leads to generation of fibronectin, which 


\section{PHASE I: INITIATION}

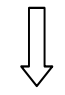

DNA and non-DNA damages

Generation of oxidative stress and reactive oxygen species

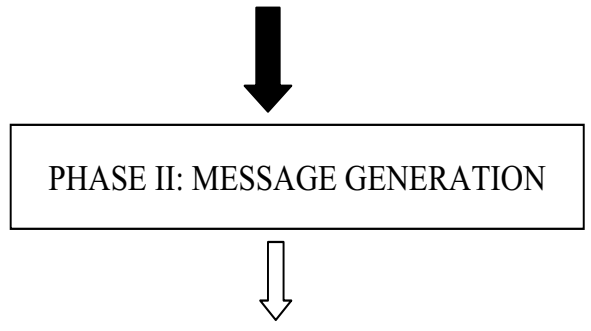

Upregulation of transcription factors (NF- $\mathrm{kB})$

Increased production of IL-1, 2 and 6

Activation of acidic sphyngomyelinases and ceramide synthase

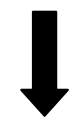

PHASE III: SIGNALING AND AMPLIFICATION

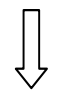

Positive feedback loops increase cytokine production

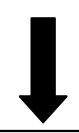

PHASE IV: ULCERATION

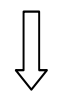

Clinically evident erosions

Bacterial colonization

Additional proinflammatory cytokine secretions

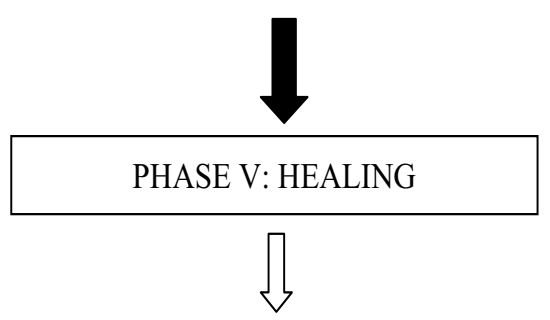

Migration of epithelial cells covering ulcerations

Mucosa appears clinically normal

Figure I Flow chart of the different phases of mucositis development. is responsible for a breakdown in connective tissue and the release of metalloproteinases responsible for more apoptosis. It should be noted that at this time the mucosa still seems clinically normal.

\section{Signaling and amplification}

Signaling and amplification is the third phase. During this period, the release of mediators from the initial injury amplifies the destruction process through activation of positive feedback loops, eg, TNF- $\alpha$ activates NF- $\kappa B$ (Sonis 2002) and sphingomyelinase (Andriea-Abadic and Levade 2002), leading to additional tissue destruction. It is interesting to note that in fractionated radiotherapy, the insult is permanent and feedback loops are constantly activated, blocking the healing process (Sonis 2004).

\section{Ulceration}

Ulceration is the fourth phase. During this period mouth injuries become clinically evident with areas of erosion. Erosions are covered by fibrinous exudates that are also called "pseudomembrane". The exudate contains oral bacteria. This period frequently coincides with the lowest neutrophil count. Bacterial colonization of the submucosa is present and activates macrophages, leading to new positive feedback and more proinflammatory cytokine secretion. Other cytokines, such as platelet-activating factor (PAF), may play an important role at this stage. It seems that platelet aggregation may worsen oral mucositis (Kuenen et al 2003). High salivary PAF levels are correlated with mucositis severity (McManus et al 1993), and pharmacologic inhibition of platelet aggregation results in reduction of mucositis (Wang et al 2002). Finally, angiogenesis is a late event of mucositis-induced ulcers.

\section{Healing}

Healing is the last phase. During this period, because of some not yet well-characterized signals, epithelial cells directly bordering the site migrate, proliferate, and differentiate. These cells move beneath the pseudomembrane to reconstitute an intact surface. This phenomenon is frequently concomitant with the return of the white blood cell count to normal values. Cells proliferate until the thickness of the mucosa is normal. However, despite the fact that the mucosa appears to be clinically normal, the environment has been altered and does not revert to its original normal state. The patient is at risk of subsequent injury with antineoplasic treatments.

Many unanswered questions remain concerning the physiopathology of oral mucositis; for instance, the role of 
saliva in the pathogenesis of oral injury is unclear. Saliva plays a major role in oral homeostasis, notably through salivary immunoglobulin A (Epstein et al 2002); however, surprisingly, an attempt to increase production by pilocarpine hydrochloride in cases of decreased or absent saliva (ie, xerostomia) failed to moderate oral mucositis (Lockhart et al 2005).

\section{Prevention and treatment of oral mucositis}

Many strategies exist to prevent and treat oral mucositis; most of these are not generally accepted and are frequently based on anecdotal reports or experiences. The lack of large, welldesigned randomized clinical studies adds to the confusion. Recently a panel of experts reviewed the oral mucositis literature and summarized the conclusions in clinical guidelines (Rubenstein et al 2004; Sonis et al 2004). Fortunately, because of a growing interest in this important side effect of cancer treatment, interesting clinical studies have been reported, and new drugs have been developed and tested.

Management of oral mucositis can be artificially divided in three basic components: general oral care, prevention, and palliative cares (Saadeh 2005).

\section{General oral care}

General oral care is the basis of oral mucositis management, and it has a crucial role in prevention of its occurrence. The aim is to reduce some host-related risk factors for stomatitis, including lowering the impact of oral microbial flora. First a pre-treatment oral assessment of the patient should be performed. Comprehensive patient education plays a central role, and a standard oral care protocol should be applied, but there is not enough evidence to recommend one protocol over another (Saadeh 2005). However, collective studies suggest that the use of a systematic protocol improves patient outcome (Rubenstein et al 2004). Some simple care should be suggested, for instance: brushing teeth twice daily using a new toothbrush at each chemotherapy cycle, daily flossing, and mouth rinsing with sterile water after each brushing or flossing. In addition, spicy food, alcoholic beverages, and alcohol-based mouthwashes should be avoided (Larson et al 1998). Topical antimicrobial treatments have frequently been shown to be ineffective in modifying mucositis (Sonis 2004). For instance use of amphotericin B, tobramycin, and polymyxin B has shown efficacy in preventing oral mucositis induced by radiotherapy but not by chemotherapy (Donnelly et al 2003). This paradox can be explained in part by the physiopathology of oral mucositis. These three compounds, in addition to their anti-infective activity, also affect the production of proinflammatory cytokines. Amphotericin B induces the expression of genes coding for TNF- $\alpha$ and IL- $1 \beta$ (Rogers et al 1998) while both tobramycin and polymyxin B attenuate the production and activity of these cytokines (Frieling et al 1997).

\section{Prevention}

Prevention is the second most important factor in addressing oral mucositis.

\section{Cryotherapy}

Cryotherapy consists of the use of ice chips when bolus 5-FU chemotherapy is administered. This cryotherapy induces a temporary vasoconstriction, reducing blood flow and chemotherapy delivery to oral mucosa (Saadeh 2005). This procedure has been validated in two randomized clinical trials (Maahood et al 1991; Cascinu et al 1994). The group receiving cryotherapy experienced a reduction in mucositis of approximately 50\% (Maahood et al 1991). In practice, ice chips are given for 30 minutes, starting 5 minutes before the beginning of the chemotherapy. It should be noted that this procedure is neither useful for continuous infusion of 5-FU nor for methotrexate and anthracycline-containing therapies since the half-life of these drugs is too long (Rubenstein et al 2004). The advantages of this procedure are its simplicity, lack of toxicity, and low cost.

\section{Low level laser therapy}

Low level laser therapy (LLLT) is also an important part of the prevention strategies of mouth mucositis (Genot and Klastersky 2005). This procedure has been shown to be effective in patients receiving HDC and HSCT (Barasch et al 1995; Cowen et al 1997). It promotes wound healing and reduces pain and inflammation (Rubenstein et al 2004). In addition, LLLT produces no toxicity and is nontraumatic to patients but requires expensive materials and is time consuming.

\section{Amifostine}

Amifostine is a radioprotectant pro-drug that when dephosphorylated protects normal but not malignant cells against oxygen-based radicals, alkylator or organoplatinum anticancer drugs (Spencer et al 2005), and radiotherapy. It acts as a free-radical scavenger and has been shown to reduce proinflammatory cytokine levels (Sonis 2004). The protection of normal cells results in part from improved vascularity, higher 
alkaline phosphatase levels (required for dephosphorylation of amifostine into its active metabolite WR-1065), and higher $\mathrm{pH}$ of normal tissues (Spencer et al 2005). One randomized trial has shown that amifostine reduced the incidence of xerostomia, but not the severity of oral mucositis after radiotherapy for head-and-neck cancers (Brizel et al 2000). A recent randomized trial suggested that for ovarian cancer patients, the frequency of severe grade III to IV carboplatinand paclitaxel-induced mucositis was reduced with amifostine infusion (Lorusso et al 2003). The study by Spencer and colleagues (2005) also suggested that amifostine reduced the median grade of oral mucositis and the severity of mucositis, but not the requirement of parenteral nutrition or analgesic use after high-dose melphalan and HSCT for multiple myeloma. Finally, amifostine reduced the frequency of severe esophagitis after chemo-radiotherapy for non-small-cell lung cancer (NSCLC) (Komaki et al 2002). Guidelines do not recommend amifostine for prevention of oral mucositis except for NSCLC patients undergoing concomitant chemo-radiotherapy (Rubenstein et al 2004). One of the main issues with amifostine administration is the toxicity. Nausea, vomiting, hypotension, sneezing, flushing, somnolence, metallic taste, allergic reactions, and hypocalcemia have been frequently reported. However, subcutaneous injection could be more tolerable (Bardet et al 2002).

\section{Chlorhexidine}

Chlorhexidine reduces mucosal colonization by microorganisms, but this effect has never been translated into clinical benefits for patients receiving either chemotherapy or conventional or high-dose radiotherapy (Saadeh 2005). In a randomized trial, chlorhexidine was found to be less effective and more toxic than a placebo rinse in patients with head-andneck tumors receiving radiotherapy (Foote et al 1994). Chlorhexidine is not recommended in the clinical guidelines (Rubenstein et al 2004). In addition, chlorhexidine has several side effects including inflammation, mouth discomfort, taste alterations, and teeth staining (Pitten et al 2003).

\section{Gelclair $^{\circledR}$}

Gelclair $^{\circledR}$ was approved by the FDA in 2002 as a class 1 medical device. It is a bioadherent gel that works by forming a coating over mucosal lesions. Gelclair consists of polyvinylpyrrolidone, hyaluronic acid, and glycerrhetinic acid. It is thought that this molecule has anti-inflammatory properties. This preparation is well tolerated and easy to use and has been shown to be effective in reducing pain and oral discomfort in patients with mucositis (Innocenti et al 2002).

\section{Benzydamine}

Benzydamine is a non-steroidal anti-inflammatory drug used topically. It also has cytoprotective, anesthetic, and antimicrobial properties (Wright et al 2005). This drug is recommended by the MASCC/ISOO panel of experts for prevention of radiotherapy-induced mucositis (Rubenstein et al 2004). This product is safe, but its efficacy for prevention of chemotherapy-induced mucositis is still unknown (Peterson et al 2004).

\section{Glutamine}

Glutamine is a neutral amino acid rich in nitrogen that plays an important role in mucosal cellular metabolism. In cancer patients treated by chemotherapy or radiotherapy, a significant glutamine depletion is frequently observed (Saadeh 2005). Conflicting results have been published about the effect of glutamine repletion in cancer patients. No positive effect was reported for patients receiving 5-FU chemotherapy (Jebb et al 1994; Okuno et al 1999); however, two smaller studies demonstrated a reduction in oral mucositis grade and duration for patients who did not receive 5-FU (Skubitz and Anderson 1996; Anderson, Schroeder, et al 1998). The effect of glutamine repletion in the BMT setting is also controversial with positive (Anderson, Ramsay, et al 1998) and negative (Pytlik et al 2002) studies.

\section{AES-I4 (Saforis ${ }^{\mathrm{TM}}$ )}

AES-14 (Saforis ${ }^{\mathrm{TM}}$ ) by Aesgen Inc., Princeton, NJ, USA, is an oral suspension delivering concentrated L-glutamine to the oral mucosa through a proprietary vehicle. This system received a fast-track approval from the FDA in January 2003 based on studies showing a reduction of mucositis severity in BMT patients and a shorter healing time after radiation (Elting et al 2004) compared with placebo. In addition AES-14 has a very favorable toxicity profile.

\section{Hematopoietic-growth factors}

It has long been observed that mucositis often resolves within days of granulocyte recovery. Some reports suggest that granulocyte colony-stimulating factor (G-CSF) or granulocytemacrophage colony-stimulating factor (GM-CSF) can reduce oral mucositis by accelerating neutrophil recovery (Wilkes 1998). However, randomized trials are controversial with positive (Chi et al 1995; Kannan et al 1997) and negative results 
(Mascarin et al 1999; Makkonen et al 2000). There is currently no evidence supporting use of G-CSF or GM-CSF subcutaneously or in the form of mouthwash for patients with oral mucositis (Karthaus et al 1998; Dazzi et al 2003). In addition, these medications are very expensive.

\section{Repifermin}

Repifermin is the human keratinocyte growth factor 2 (KGF2). This substance has been studied in a phase II trial in which patients received conditioning chemotherapy before autologous HSCT. Repifermin significantly decreased the incidence of grade 2 to 4 mucositis (Freytes et al 2004).

\section{Palifermin}

Palifermin is the recombinant keratinocyte growth factor 1 (rHuKGF-1). This molecule was purified from fibroblast culture fluid in 1989 as a protein of 26-28kDa (Rubin et al 1989). Cloning showed that KGF and its receptor are members of the fibroblast growth factor (FGF) family with $30 \%-45 \%$ homology with other proteins of the FGF family (Finch et al 1989). It is also called FGF-7. Keratinocyte growth factor is produced by fibroblasts and microvascular endothelial cells in different organs like lungs, skin, stomach, and bladder (MacDonald and Hill 2002). Keratinocyte growth factor receptor expression has been found in gut epithelium, hepatocytes, skin keratinocytes, alveolar type II cells, mammary epithelium, and urothelium. Keratinocyte growth factor is also a mediator of hair follicle growth and development (Oelmann et al 2004). It can be considered as a mesenchymally-derived paracrine mediator of epithelial growth and development (MacDonald and Hill 2002).

Like other members of the FGF family, KGF is a heparinbinding molecule and heparin is required for maximal KGF stimulation through its receptor (Hsu et al 1999). When bound to the receptor, KGF is internalized by endocytosis (MacDonald and Hill 2002). Keratinocyte growth factor expression seems mediated by pro-inflammatory cytokines like TNF- $\alpha$, IL-1, and IL-6 (Chedid et al 1994). Glucocorticoids appear to decrease KGF production (impairing wound healing) while estrogen and progesterone increase KGF in mammary gland and endometrium (MacDonald and Hill 2002). Beneficial effects of KGF on mucosa seem to be broader than expected. For instance, KGF upregulates the transcription factor Nrf 2 that encodes genes playing a role in detoxifying radical oxygen species (Braun et al 2002; auf dem Keller et al 2004), attenuating TNF- $\alpha$, and in the expression of adhesion molecules (Just et al 2003).
One major concern about KGF is the presence of the receptor on malignant cells from a variety of tissues (Oelmann et al 2004). This finding has raised the possibility that exogenous KGF might stimulate tumor cells. Interestingly, some authors observed increased expression of the $\mathrm{KGF} / \mathrm{KGF}$ receptor in more-differentiated and less-advanced tumors, and absence of expression in malignant versus benign cells or a switch to FGF receptor isoforms with low binding affinity to KGF (Oelmann et al 2004). In addition, KGF has been described as stimulating matrix metalloproteinases and inducing angiogenesis, mechanisms related to tumor progression and metastatic potential. Fortunately, several reports have shown that even if KGF modestly stimulated tumor cell growth for a minority of solid tumor samples tested, it did not impair tumor sensitivity to chemotherapy (Oelmann et al 2004) or radiotherapy (Hille et al 2003).

Pharmacokinetic variables of palifermin have been determined in healthy volunteers and in metastatic colorectal cancer patients (Neumann and Foote 2003). Palifermin has been tested in clinical trials. In a phase I trial, Meropol determined that KGF was well tolerated at doses up to $40 \mu \mathrm{g} /$ $\mathrm{kg}$ /day administered IV for 3 days before 5-FU plus leucovorin in patients with advanced colon cancers (Meropol et al 2003). This phase I study was randomized and suggested that patients treated with palifermin had a lower rate of grade 2 to 4 mucositis compared with patients who received placebo. It has been shown recently in a large randomized trial that palifermin reduced the incidence of grade 3 to 4 mucositis, decreased the median duration of mucositis, the duration of opioid analgesic use, and the incidence of use of parenteral nutrition in patients with hematologic cancers receiving total-body irradiation and HDC (Spielberger et al 2004). Adverse events encountered were mild to moderate and transient and consisted mainly of rash, pruritus, erythema, edema, hypoesthesia, paresthesia, and mouth and tongue disorders like thickening and taste (Meropol et al 2003). Asymptomatic and reversible increases of amylases and lipases have also been observed (Sharma et al 2005).

In December 2004, palifermin was approved by the FDA for prevention of mucositis in patients receiving $\mathrm{HDC}$ and HSCT (Saadeh 2005). This molecule is the first innovative "target-based" biologic agent for prevention of oral mucositis. Many trials are currently ongoing to determine the efficacy of this molecule in patients receiving radiotherapy, various chemotherapy regimens, or an allogenic transplant. In animal models, however, it has been shown that KGF can reduce acute oral and intestinal mucositis after chemoradiotherapy or 
fractionated irradiation (Farell et al 1998; Dörr et al 2002). Recently in allogeneic and haploidentical bone marrow transplant animal models, KGF has shown interesting properties in reducing the incidence of graft-versus-host disease (GvHD). This effect seemed to be independent of repair of conditioninginduced injuries (Panoskaltsis-Mortari, Taylor, et al 2000); Vanclée et al 2005). In addition, it has been demonstrated that KGF has a favorable effect on lung repair in mice after allogeneic bone marrow transplant (Panoskaltsis-Mortari, Ingbar, et al 2000). A recent clinical study reported 69 patients who received palifermin after high-dose chemo-radiotherapy and allogeneic bone marrow transplantation. This medication was shown to be safe and well tolerated and did not have any negative effects on engraftment, GvHD, and survival (Blazar et al 2005). Another clinical study even suggested a survival benefit in patients with a high risk for acute GvHD when palifermin was added to tacrolimus and methotrexate (Reynolds et al 2004).

\section{Palliative cares}

Palliative cares are implemented when mucositis is present. Continuation of routine oral care should be advised (Wright et al 2005). In addition, dietary guidelines should be respected with avoidance of tobacco, alcohol, and spicy food. In some cases, parenteral or enteral supports may even be required. Finally, correct analgesia should be implemented. Morphine has been recommended as the treatment of choice for patients with mucositis undergoing HDC and HSCT (Rubenstein et al 2004). Prevailing clinical guidelines can help the clinician to choose to best analgesia for the patient (WHO 1996). These guidelines include the use of opioids, nonopioids, adjuvant medications, and assessment tools. Topical anesthetics such as viscous lidocaine are frequently used to decrease pain related to oral mucositis; however, there are no experimental studies to support this approach. In addition, viscous lidocaine requires frequent applications and can be accompanied by burning sensation and the blunting of taste (Saadeh 2005). A summary of available therapeutic and prophylactic interventions is presented in Table 2.

\section{Conclusions}

Oral mucositis is a frequent complication of anticancer treatments that has long been overlooked and underreported. The discrepancies between the different scales used to assess the severity of mucositis add more confusion, and large randomized clinical studies are scarce. Recently there has been greater focus on the frequency, physiopathology, consequences, and treatments of oral mucositis. These treatments remain largely based on anecdotal reports and assumptions, but some interesting studies have been done to provide evidence for these approaches. The understanding of the physiopathological basis of mucositis led to the development of target drug therapies, of which palifermin is the hallmark. This new drug constitutes a milestone in the management of cancer treatment-induced toxicity. Other molecules are currently under investigation, including transforming growth factor- $\beta 3$, IL- 11 , and FGF-20 (Peterson and Cariello 2004).

The physiopathology of mucositis can also explain why some preventive treatments like topical anti-infective agents may have some paradoxical effects. Guidelines have recently been developed to help clinicians in the daily management of this devastating toxicity (Rubenstein et al 2004; Sonis et al 2004). One can not insist enough on the importance of prevention, systematic oral care, and good patient information. When oral mucositis is profuse, appropriate analgesia is required. It is clear that treatment of mucositis is not based on a unique and non-existent magic drug, but rather on a logical and rational use of different modalities. Combining drugs in specific sequences and at different times relative to the administration of anticancer treatment will probably be the solution for adequate management of oral mucositis. In the near future, dose, timing, and sequencing of anti-mucositis agents will probably be as well developed as chemotherapy regimens (Peterson and Cariello 2004). It is interesting to note that new drugs in development seem to have different mechanisms of action. In addition, it is no longer pure optimism to expect effective specific treatments of mucositis in the near future (Sonis 2004).

Table 2 Candidate interventions for the management of oral mucositis

\begin{tabular}{|c|c|c|}
\hline General oral care & Prevention & $\begin{array}{l}\text { Palliative } \\
\text { cares }\end{array}$ \\
\hline $\begin{array}{l}\text { Comprehensive patient } \\
\text { education }\end{array}$ & Cryotherapy & $\begin{array}{l}\text { Continuation of } \\
\text { routine oral cares }\end{array}$ \\
\hline Standard oral care protocol & $\begin{array}{l}\text { Low-level laser } \\
\text { therapy }\end{array}$ & $\begin{array}{l}\text { Dietary guidelines: } \\
\text { avoidance of } \\
\text { alcohol, tobacco, } \\
\text { spicy food }\end{array}$ \\
\hline Brushing teeth twice daily & Amifostine & $\begin{array}{l}\text { Enteral or } \\
\text { parenteral support }\end{array}$ \\
\hline Daily flossing & Gelclair $^{\circledR}$ & Correct analgesia \\
\hline $\begin{array}{l}\text { Mouth rinsing with sterile } \\
\text { water }\end{array}$ & Benzydamine & \\
\hline \multirow[t]{2}{*}{$\begin{array}{l}\text { Avoidance of spicy food, } \\
\text { alcohol, tobacco }\end{array}$} & AES-I4 & \\
\hline & Palifermin & \\
\hline
\end{tabular}




\section{References}

Anderson PM, Schroeder G, Skubitz KM. 1998. Oral glutamine reduces the duration and severity of stomatitis after cytotoxic cancer chemotherapy. Cancer, 83:1433-9.

Anderson PM, Ramsay NK, Shu XO, et al. 1998. Effect of low-dose oral glutamine on painful stomatitis during bone marrow transplantation. Bone Marrow Transplant, 22:339-44.

Andriea-Abadic N, Levade T. 2002. Sphingomyelin hydrolysis during apoptosis. Biochem Biophys Acta, 1585:126-34.

auf dem Keller U, Krampert M, Kümin A, et al. 2004 Keratinocyte growth factor: effects on keratinocytes and mechanisms of action. Eur J Biol, 83:607-12.

Balducci L, Exterrman M. 2000. Management of cancer in the older person: a practical approach. Oncologist, 20:224-37.

Barasch A, Peterson DE, Tanzer JM, et al. 1995. Heliun-neon laser effects on conditioning-induced oral mucositis in bone marrow transplantation patients. Cancer, 76:2550-6.

Bardet E, Martin L, Calais G, et al. 2002. Preliminary data of the GORTEC 2000-02 phase III trial comparing intravenous and subcutaneous administration of amifostine for head and neck tumors treated by external radiotherapy. Sem Oncol, 29(suppl 19):57-60.

Bellm LA, Epstein JB, Rose-Ped A, et al. 2000. Patients reports of complications of bone marrow transplantation. Support Care Cancer, 8:33-9.

Blazar BR, Weisdorf DJ, DeFor TE, et al. 2005. Palifermin (A rHuKGF molecule) is safe and well tolerated in patients (Pts) with hematologic malignancies (HM) undergoing high-dose chemoradiotherapy (HD-CRT) followed by allogeneic hematopoietic stem cell transplant (HSCT) [abstract]. Biol Blood Marrow Transplant, 11(suppl 1):11.

Braun S, Hanselmann C, Gassman MG, et al. 2002. Nrf2 transcription factor: a novel target of keratinocyte growth factor action regulates gene expression and inflammation in the healing skin wound. Mol Cell Biol, 22:5492-505.

Brizel DM, Wasserman TH, Henke M, et al. 2000. Phase III randomised trial of amifostine as a radioprotector in head and neck cancer. J Clin Oncol, 18:3339-45.

Cascinu S, Fedeli A, Fedeli SL, et al. 1994. Oral cooling (cryotherapy), an effective treatment for the prevention of 5-fluorouracil-induced stomatatis. Eur J Cancer B Oral Oncol, 30B:234-6.

Chedid M, Rubin JS, Csaky KG, et al. 1994. Regulation of keratinocyte growth factor gene expression by interleukin 1. J Biol Chem, 269:10753-7.

Chi KH, Chen CH, Chan WK, et al. 1995. Effect of granulocyte-macrophage colony-stimulating factor on oral mucositisin head and neck cancer patients after cisplatin, fluorouracil, and leucovorin chemotherapy. $J$ Clin Oncol, 13:2620-8.

Cowen D, Tardieu C, Schubert M, et al. 1997. Low energy helium-neon laser in the prevention of oral mucositis in patients undergoing bone marrow transplan: results of a double blind randomised trial. Int J Radiot Oncol Biol Phys, 38:697-703.

Dazzi C, Cariello A, Giovanis P, et al. 2003. Prophylaxis with GM-CSF mouthwashes does not reduce frequency and duration of severe oral mucositis in patients with solid tumors undergoing high-dose chemotherapy with autologous peripheral blood stem cell transplantation rescue: a double blind randomised, placebo-controlled study. Ann Oncol, 14:559-63.

Donnelly JP, Bellm LA, Epstein JB, et al. 2003. Antimicrobial therapy to prevent or treat mucositis. Lancet Infect Dis, 3:405-12.

Dood MJ, Miaskowski C, Shiba GH, et al. 1999. Risk factors for chemotherapy-induced oral mucositis: dental appliance, oral hygiene, previous oral lesions, and a history of smoking. Cancer Invest, 17:27884.

Dörr W, Spekl K, Farell C. 2002. Amelioration of acute oral mucositis by keratinocyte growth factor: fractionated irradiation. Int $J$ Radiat Oncol Biol Phys, 54:245-51.
Elting L, Peterson D, Sonis ST. 2004. Late-breaking information from the 2004 ASCO annual meeting. Oral Mucositis Newsletter, 1:1-12.

Epstein JB, Tsang AHF, Warkentin D, et al. 2002. The role of salivary function in modulating chemotherapy-induced oropharyngeal mucositis: a review of the literature. Oral Surg Oral Med Oral Pathol Oral Radiol Endod, 94:39-44.

Farell CL, Bready JV, Rex KL, et al. 1998. Keratinocyte Growth Factor protects mice from chemotherapy and radiation-induced gastrointestinal injury and mortality. Cancer Res, 58:933-9.

Finch PW, Rubin JS, Miki T, et al. 1989. Human KGF is FGF-related with properties of a paracrine effector of epithelial cell growth. Science, 245:752-5.

Foote RL, Loprinzi CL, Frank AR et al. 1994. Randomized trial of chlorhexidine mouthwash for alleviation of radiation induced-mucositis. J Clin Oncol, 12:2630-3.

Freytes CO, Ratanathorn V, Taylor C, et al. 2004. Phase I/II randomised clinical trial evaluating the safety and clinical effects of repifermin administered to reduce mucositis in patients undergoing autologous hemotopoietic stem cell transplantation. Clin Cancer Res, 10:8318-24.

Frieling JT, Mulder JA, Hendriks T, et al. 1997. Differential induction of proand anti-inflammatory cytokines in whole blood by bacteria effects of antibiotic treatment. Antimicrob Agents Chemother, 41:1439-43.

Genot MT, Klastersky J. 2005. Low level laser for prevention and therapy of oral mucositis induced by chemotherapy and/or radiotherapy. Curr Opin Oncol, 17:236-40.

Graham GJ, Ramenghi U, O'Connor MP, et al. 1992. Studies of oral neutrophil levels in patients receiving G-CSF after autologous marrow transplantation. Br J Haematol, 82:589-95.

Henry S, D’Hondt L, André M, et al. 2004. Saccharomyces Cerevisiae fumgemia in a head and neck cancer patient: a case report and review of the literature. Acta Clin Belg, 59:220-2.

Hille A, Rave-Frank M, Pradier O, et al. 2003. Effect of keratinocyte growth factor on the proliferatio, clonogenic capacity and colony size of human epithelial tumour cells in vitro. Int J Radiat Biol, 79:119-128.

Hsu YR, Nybo R, Sullivan JK, et al. 1999. Heparin is essential for a single keratinocyte growth factor molecule to bind and form a complex with two molecules of the extracellular domain of its receptor. Biochemistry, 38:2523-34

Innocenti M, Moscatelli G, Lopez S. 2002. Efficacy of Gelclair in reducing pain in palliative care patients with oral lesions: preliminary findings from an open pilot study. J Pain Symptom Manage, 24:456-7.

Jebb SA, Osborne RJ, Maughan TS, et al. 1994. 5-fluorouracil and folinic acid-induced mucositis: no effect of oral glutamine supplementation. $\mathrm{Br}$ J Cancer, 70:732-5.

Just N, Tillie-Leblond I, Guery BP, et al. 2003. Keratinocyte growth factor (KGF) decreases ICAM-1 and VCAM-1 cell expression on bronchial epithelial cells. Clin Exp Immunol, 132:61-9.

Kannan V, Bapsy PP, Anantha N et al. 1997. Efficacy and safety of granulocytemacrophage colony-stimulating factor (GM-CSF) on the frequency and severity of radiation mucositis in patients with head and neck carcinoma. Int J Radiot Oncol Biol Phys, 37:1005-10.

Karthaus M, Rosenthal C, Huebner G, et al. 1998. Effect of topical oral GCSF on oral mucositis: a randomized placebo-controlled trial. Bone Marrow Transplant, 22:781-5.

Komaki R, Lee JS, Kaplan B et al. 2002. Randomised phase III study of chemoradiation with or without amifostine for patients with favourable performance status inoperable stage II and III non-small cell lung cancer: preliminary results. Semin Radiot Oncol, 12(suppl 1):46-9.

Kuenen BC, Levi M, Meijers JC, et al. 2003. Potential role of platelets in endothelial damage during treatment with gemcitabine and the angiogenesis inhibitor SU5416. J Clin Oncol, 21:2192-8.

Larson P, Miakowski C, MacPhail L, et al. 1998. The PRO-SELF mouth aware program: an effective approach for reducing chemotherapy-induced mucositis. Cancer Nurs, 21:263-8. 
Lockhart PB, Brennan MT, Kent ML, et al. 2005. Randomized controlled trial of pilocarpine hydrochloride for the moderation of oral mucositis during autologous blood stem cell transplantation. Bone Marrow Transplant, 35:713-20.

Lorusso D, Ferrandina G, Greggi S, et al. 2003. Phase III multicenter randomised trial of amifostine as cytoprotectant in first-line chemotherapy in ovarian cancer patients. Ann Oncol, 14:1086-93.

MacDonald K, Hill G. 2002. Keratinocyte growth factor (KGF) in haematology and oncology. Curr Pharm Des, 8:395-403.

Mahood DJ, Dose AM, Loprinzi CL, et al.1991. Inhibition of fluouracilinduced stomatatis by oral cryotherapy. J Clin Oncol, 9:449-52.

Makkonen TA, Minn H, Jekunen A, et al. 2000. Granulocyte macrophagecolony stimulating factor (GM-CSF) and sucralfate in prevention of radiation-induced mucositis: a prospective randomised study. Int J Radiat Oncol Biol Phys, 46:525-34.

Mascarin M, Franchin G, Minatel E, et al. 1999. The effect of granulocyte colonystimulating factor on oral mucositisin head and neck cancer patients treated with hyperfractionated radiotherapy. Oral Oncol, 35:203-8.

McManus LM, Ostrom KK, Laer C, et al. 1993. Radiation-induced increased platelet-activating factor activity in mixed saliva. Lab Invest, 68:118-24.

[MAC] Meta-Analysis Group in Cancer. 1998. Toxicity of fluorouracil in patients with advanced colorectal cancer: effect of administration schedule and prognostic factors. Meta-Analysis Group in Cancer. J Clin Oncol, $16: 3537-41$

Meropol NJ, Somer RA, Gutheil J, et al. 2003. Randomized phase I trial of recombinant human keratinocyte growth factor plus chemotherapy: potential role as mucosal protectant. J Clin Oncol, 21:1452-8.

Neumann TK, Foote MA. 2003. The development of supportive-care agents for patients with cancer. Biotechn Ann Rev, 9:397-416.

Oelmann E, Haghu S, Kulimova E, et al. 2004. Influence of keratinocyte growth factor on clonal growth of epithelial cells, lymphoma and leukaemia cells and on sensitivity of tumor cells towards 5-fluorouracil in vitro. Int J Oncol, 25:1001-12.

Okuno SH, Woodhouse CO, Loprinzi CL, et al. 1999. Phase III controlled evaluation of glutamine for decreasing stomatitis in patients receiving fluorouracil (5-FU)-based chemotherapy. Am J Clin Oncol, 22:258-61.

Panoskaltsis-Mortari A, Taylor PA, Rubin JS, et al. 2000. Keratinocyte growth factor facilitates alloengraftment and ameliorates graft-versus-host disease in mice by a mechanism independent of repair of conditioning-induced tissue injury. Blood, 96:4350-6.

Panoskaltsis-Mortari A, Ingbar DH, Jung P, etal. 2000. KGF pretreatment decreases B7 and granzyme B expression and hastens repair in lungs of mice after allogeneic BMT. Am J Physiol Lung Cell Mol Physiol, 278:988-99.

Peterman A, Cella D, Glandon G, et al. 2001. Mucositis in head and neck cancer : economic and quality-of-life outcomes. J Natl Cancer Inst Monogr, 29:45-51.

Peterson DE, Cariello A. 2004. Mucosal damage: a major risk factor for severe complications after cytotoxic therapy. Sem Oncol, 31:35-44.

Peterson D, Beck S, Keefe D. 2004. Novel therapies. Sem Oncol Nursing, 20:53-8.

Pitten FA, Kiefer T, Buth C, et al. 2003. Do cancer patients with chemotherapyinduced leukopenia benefit from an antiseptic chlorhexidine-based oral rinse? A double-blind, block-randomised, controlled study. J Hosp Infect, 53:283-91

Popescu RA, Norman A, Ross PJ, et al. 1999. Adjuvant or palliative chemotherapy for colorectal cancerin patients 70 years or older. $J$ Clin Oncol, 17:2412-8.

Pytlik R, Benes P, Patorkova M et al. 2002. Standardized parenteral alanylglutamine dipeptide supplementation is not beneficial in autologous transplant patients: a randomised, double-blind placebo controlled study. Bone Marrow Transplant, 30:953-61.

Rao Naidu MU, Venkat Ramana G, Usha Rani P, et al. 2004. Chemotherapyinduced and/or radiation therapy-induced oral mucositis-complicating the treatment of cancer. Neoplasia, 6:423-31.
Reynolds CM, Levine JE, Uberti JP, et al. 2004. Keratinocyte Growth Factor (Palifermin) in combination with tacrolimus and methotrexate for the prevention of acute graft-vs-host disease (aGvHD) in patients at high risk of a GvHD [abstract]. Blood, 104:1228.

Rogers PD, Jenkins JK, Chapman SW, et al. 1998. Amphotericin B activation of human genes encoding for cytokines. $J$ Infect Dis, 178:1726-33.

Rubenstein EB, Peterson DE, Schubert M, et al. 2004. Clinical practice guidelines for the prevention and treatment of cancer therapy-induced oral and gastrointestinal mucositis. Cancer, 100:2026-46.

Rubin JS, Osada H, Finch PW, et al. 1989. Purification and characterization of a newly identified growth factor specific for epithelial cells. Proc Natl Acad Sci U S A, 86:802-6.

Saadeh CE. 2005. Chemotherapy and radiotherapy-induced oral mucositis: review of perspective strategies and treatment. Pharmacotherapy, 25:540-54.

Sharma R, Tobin P, Clarke SJ. 2005. Management of chemotherapy-induced nausea, vomiting, oral mucositis, and diarrhoea. Lancet Oncol, 6:93-102.

Skubitz KM, Anderson PM. 1996. Oral glutamine to prevent chemotherapy induced stomatatis: a pilot study. J Lab Clin Med, 127:223-8.

Sonis ST, Elting LS, Keefe D, et al. 2004. Perspectives on cancer therapyinduced mucosal injury. Pathogenesis, measurement, epidemiology, and consequences for patients. Cancer, 100:1995-2025.

Sonis ST. 2004. A biological approach to mucositis. J Support Oncol, 2:2136.

Sonis ST. 1998. Mucositis as a biologic process: a new hypothesis for the development of chemotherapy-induced stomatotoxicity. Oral Oncol, 34:39-43.

Sonis ST. 2002. The biologic role for nuclear factor-kappaB in disease and its potential involvement in mucosal injury associated with anti-neoplasic therapy. Crit Rev Oral Biol Med, 13:380-9.

Spencer A, Horvath N, Gibson J, et al. 2005. Prospective randomised trial of amifostine cytoprotection in myeloma patients undergoing high-dose melphalan conditioned autologous stem cell transplantation. Bone Marrow Transplant, 35:971-7.

Spielberger R, Stiff P, Bensinger W, et al. 2004. Palifermin for oral mucositis after intensive therapy for hematologic cancers. $N$ Engl J Med, 351:10-18.

Trotti A, Byhardt R, Stetz J, et al. 2000. Common toxicity criteria: version 2.0. An improved reference for grading the acute effects of cancer treatment: impact on radiotherapy. Int J Radiat Oncol Biol Phys, 47:13-47.

Ulrich CM, Yasui Y, Storb R, et al. 2001. Pharmacogenetics of methotrexate toxicity among marrow transplantation patients varies with the methylenetetrahydrofolate reductase C677T polymorphism. Blood, 98:231-4.

Vanclée A, Lutgens LC, Oving EB, et al. 2005. Keratinocyte growth factor ameliorates acute graft-versus-host disease in a novel nonmyeloablative haploidentical transplantation model. Bone Marrow Transplant, 36:907-15.

Wang J, Albertson CM, Zheng H, et al. 2002. Short-term inhibition of ADPinduced platelet aggregation by clopidrogel ameliorates radiation-induced toxicity in rat small intestine. Thromb Haemost, 87:122-8.

Wardley AM, Jayson GC, Swindell R, et al. 2000. Prospective evaluation of oral mucositis in patients receiving myeloablative conditioning regimens and hematopoietic progenitor rescue. Br J Haematol, 110:292-9.

Wilkes JD. 1998. Prevention and treatment of oral mucositis following cancer chemotherapy. Sem Oncol, 25:538-51.

[WHO] World Health Organization. 1996. Cancer pain relief(2nd ed). Genova, Italy: WHO

Wright J, Feld R, Knox J. 2005. Chemotherapy-induced oral mucositis: new approaches to prevention and management. Exp Opin Drug Saf, 4:193200.

Wymenga AN, van der Graaf WT, Spijkervet FK, et al. 1997. A new in vitro assay of quantitation of chemotherapy induced mucositis. $\mathrm{Br}$ J Cancer, 8:1062-6.

Wymenga AN, van der Graaf WT, Hofstra LS, et al. 1999. Phase I study of transforming growth factor-beta 3 mouthwashes for prevention of chemotherapy induced mucositis. Clin Cancer Res, 5:1363-8. 